\title{
Asymptotic tail probability of weighted infinite sum of conditionally dependent and consistently varying tailed random variables
}

Qingwu GaO ${ }^{1,2^{*}+}$, Xijun Liu ${ }^{3+}$ and Chunhong Chai ${ }^{3}$

\author{
"Correspondence: \\ qwgao@aliyun.com; \\ qingwuga@buffalo.edu \\ 'School of Statistics and \\ Mathematics, Nanjing Audit \\ University, Nanjing, China \\ ${ }^{2}$ Department of Industrial and \\ Systems Engineering, University at \\ Buffalo, Buffalo, USA \\ Full list of author information is \\ available at the end of the article \\ ${ }^{\dagger}$ Equal contributors
}

\begin{abstract}
This paper investigates the asymptotic behavior of the tail probability of a weighted infinite sum of random variables with consistently varying tails under two conditional dependence structures. The obtained results extend and improve the existing results of Bae and Ko (J. Korean Stat. Soc. 46:321-327, 2017).
\end{abstract}

MSC: Primary 62E20; secondary 60G70; 62H2O

Keywords: Asymptotic tail probability; Weighted infinite sum; Conditional dependence; Consistent variation

\section{Introduction}

Assume that $\left\{X_{i}, i \geq 1\right\}$ is a sequence of random variables (r.v.s) with their respective distributions $F_{i}, i \geq 1$, supported on $D=[0, \infty)$ or $(-\infty, \infty)$, and that $\left\{\psi_{i}, i \geq 1\right\}$ is a sequence of real numbers, which represent the weights of $\left\{X_{i}, i \geq 1\right\}$. Denote the weighted infinite sum by $\sum_{i=1}^{\infty} \psi_{i} X_{i}$, the asymptotic tail behavior of which is the main objective of our paper.

In this paper, we consider the heavy-tailed distribution classes. Firstly, we introduce some notions and notations. All limit relationships henceforth hold as $x \rightarrow \infty$ unless stated otherwise. For two positive functions $a(\cdot)$ and $b(\cdot)$, we write $a(x) \lesssim b(x)$ if $\lim \sup a(x) / b(x) \leq 1, a(x) \gtrsim b(x)$ if $\liminf a(x) / b(x) \geq 1, a(x) \sim b(x)$ if $\lim a(x) / b(x)=1$. For a proper distribution $V$ on $(-\infty, \infty)$, we denote its tail by $\bar{V}(x)=1-V(x)$, and its upper and lower Matuszewska indices, respectively, by

$$
J_{V}^{+}=\inf \left\{-\frac{\log \bar{V}_{*}(y)}{\log y}: y>1\right\} \text { and } J_{V}^{-}=\sup \left\{-\frac{\log \bar{V}^{*}(y)}{\log y}: y>1\right\}
$$

where $\bar{V}_{*}(y)=\liminf \bar{V}(x y) / \bar{V}(x)$ and $\bar{V}^{*}(y)=\limsup \bar{V}(x y) / \bar{V}(x)$ for $y>0$.

An important class of heavy-tailed distributions is the subexponential class. Say that a distribution $V$ on $[0, \infty)$ belongs to the subexponential class, denoted by $V \in \mathscr{S}$, if

$$
\overline{V^{* 2}}(x) \sim \bar{V}(x)
$$

\section{空 Springer}

(c) The Author(s) 2019. This article is distributed under the terms of the Creative Commons Attribution 4.0 International License (http://creativecommons.org/licenses/by/4.0/), which permits unrestricted use, distribution, and reproduction in any medium, provided you give appropriate credit to the original author(s) and the source, provide a link to the Creative Commons license, and indicate if changes were made. 
where $V^{* 2}$ is the 2-fold convolution of $V$. Note that if $V \in \mathscr{S}$ then $V$ is long-tailed, denoted by $V \in \mathscr{L}$, in the sense that

$$
\bar{V}(x+y) \sim \bar{V}(x), \quad \text { for any } y>0 .
$$

Besides, if $V \in \mathscr{L}$ then

$$
\begin{aligned}
\mathscr{H}(V)= & \{h: x \in[0, \infty), h(x) \uparrow \infty, h(x)=o(1) x \text { and } \bar{V}(x+y) \sim \bar{V}(x) \text { holds uniformly } \\
& \text { for all }|y| \leq h(x)\} \\
& \neq \varnothing .
\end{aligned}
$$

Moreover, the class $\mathscr{S}$ covers the class $\mathscr{C}$ of distributions with consistently varying tails, characterized by

$$
\lim _{y \downarrow 1} \bar{V}_{*}(y)=1, \quad \text { or equivalently, } \quad \lim _{y \uparrow 1} \bar{V}^{*}(y)=1 ;
$$

and also the class $\mathscr{C}$ covers the class $\mathscr{R}_{-\alpha}, 0<\alpha<\infty$, of distributions with regularly varying tails, characterized by

$$
\bar{V}(x y) \sim y^{-\alpha} \bar{V}(x) .
$$

Another important class of heavy-tailed distributions is the dominant variation class, denoted by $\mathscr{D}$. Say that a distribution $V$ belongs to the class $\mathscr{D}$, if

$$
\bar{V}^{*}(y)<\infty, \quad \text { for any } y>0 .
$$

More generally, when $V$ is supported on $(-\infty, \infty)$, we say that $V$ belongs to a distribution class if $V(x) 1_{\{x \geq 0\}}$ belongs to the class. In conclusion,

$$
\mathscr{R}_{-\alpha} \subset \mathscr{C} \subset \mathscr{L} \cap \mathscr{D} \subset \mathscr{S} \subset \mathscr{L}
$$

For more details of heavy-tailed distributions and their applications, the reader is referred to Bingham et al. [2] and Embrechts et al. [5].

By inequality (2.1.9) in Theorem 2.18 and Proposition 2.2.1 of Bingham et al. [2], we know that $V \in \mathscr{D}$ if and only if $J_{V}^{+}<\infty$; and if $V \in \mathscr{D}$, then, for all $0<p_{1}<J_{V}^{-}$and $p_{2}>J_{V}^{+}$, there exist $C_{i}>0$ and $D_{i}>0, i=1,2$ such that

$$
\frac{\bar{V}(x y)}{\bar{V}(x)} \leq C_{1} y^{-p_{1}}, \quad x y \geq x \geq D_{1}
$$

and

$$
\frac{\bar{V}(x)}{\bar{V}(x y)} \leq C_{2} y^{p_{2}}, \quad x y \geq x \geq D_{2} .
$$

We now give a proposition, which will play a key role in the proofs of the main results. 
Proposition 1.1 If $V \in \mathscr{C}$, then $J_{V}^{-}>0$.

Proof For any fixed $x>0, \bar{V}(x y) / \bar{V}(x)$ is a monotonically decreasing function of $y$, which leads to $\bar{V}^{*}(y) \leq \bar{V}^{*}(z)$ for $y>z>0$, and then by $V \in \mathscr{C}, \bar{V}^{*}(y) \leq \lim _{z \uparrow 1} \bar{V}^{*}(z)=1$. Since $\lim \sup _{x \rightarrow \infty} \lim _{y \rightarrow \infty} \bar{V}(x y) / \bar{V}(x)=0$, there exists a sufficiently large number $y_{0}>1$ such that $\bar{V}^{*}(y)<1$ for all $y>y_{0}$, and further $\log \bar{V}^{*}(y) / \log y<0, y>y_{0}>1$. Hence by the definition of $J_{V}^{-}$, it follows that $J_{V}^{-} \geq \sup \left\{-\log \bar{V}^{*}(y) / \log y: y>y_{0}\right\}>0$.

It is well known that an increasing number of researchers introduce many dependence structures to extensively study the asymptotic tail behaviors of sums of r.v.s in the literature of applied probability. See, for example, Ko and Tang [14], Geluk and Tang [12], Chen and Yuan [4], Foss and Richards [6], Gao and Wang [10], Yi et al. [21], Liu et al. [17], Gao and Liu [9], Chen et al. [3], Li [15], Wang et al. [20], Jiang et al. [13], Gao and Yang [11], Gao and Jin [8], Liu et al. [16, 18], Bae and Ko [1], Gao et al. [7], among which Ko and Tang [14] proposed a conditional dependence structure as follows.

Assumption A For $n \geq 2$ and $D=[0, \infty)$, there exist some large constants $x_{0}=x_{0}(n)>0$ and $C=C(n)>0$ such that, for every $2 \leq j \leq n$,

$$
\limsup \sup _{x_{0} \leq t \leq x-x_{0}} \frac{P\left(X_{1}+\cdots+X_{j-1}>x-t \mid X_{j}=t\right)}{P\left(X_{1}+\cdots+X_{j-1}>x-t\right)} \leq C .
$$

In this paper, we extend the support of corresponding distribution in Assumption A from $[0, \infty)$ to $(-\infty, \infty)$, and we denote by Assumption $A^{*}$ the modified dependence structure.

Besides, Geluk and Tang [12] introduced another conditional dependence structure.

Assumption B For $n \geq 2$ and $D=(-\infty, \infty)$, there exist some large constants $x_{0}=x_{0}(n)>0$ and $C=C(n)>0$ such that the inequality

$$
P\left(\left|X_{i}\right|>x_{i} \mid X_{j}=x_{j} \text { with } j \in J\right) \leq C \overline{F_{i}}\left(x_{i}\right)
$$

holds for all $1 \leq i \leq n, J:=\{j: 1 \leq j \leq n\} \backslash\{i\}, x_{i}>x_{0}$, and $x_{j}>x_{0}, j \in J$.

Obviously, the relation in Assumption B is equivalent to the conjunction of the relations

$$
P\left(X_{i}>x_{i} \mid X_{j}=x_{j} \text { with } j \in J\right) \leq C \overline{F_{i}}\left(x_{i}\right)
$$

and

$$
P\left(X_{i}<-x_{i} \mid X_{j}=x_{j} \text { with } j \in J\right) \leq C \overline{F_{i}}\left(x_{i}\right)
$$

In this paper, for Assumption B, relation (1.3) is replaced by the following relation:

$$
P\left(X_{i}<-x_{i} \mid X_{j}=x_{j} \text { with } j \in J\right) \leq C F_{i}\left(-x_{i}\right)
$$

to cover all independent r.v.s In fact, when $\left\{X_{i}, 1 \leq i \leq n\right\}$ is a sequence of mutually independent r.v.s such that $\lim _{x_{i} \rightarrow \infty} \overline{F_{i}}\left(x_{i}\right) / F_{i}\left(-x_{i}\right)=0$ for some $1 \leq i \leq n$, relation (1.3) is not 
satisfied, and then neither is Assumption B. Hence, the extended conditional dependence structure from Assumption B is labeled as Assumption $B^{*}$. Note that these extended conditional dependence structures denoted by Assumptions $\mathrm{A}^{*}$ and $\mathrm{B}^{*}$ were firstly considered by Jiang et al. [13].

This paper will mainly focus on the asymptotic behavior of the tail probability of a weighted infinite sum of heavy-tailed r.v.s under the above two extended conditional dependence structures. In the rest of this paper, we will state our main results in Sect. 2, and prove them in Sect. 3.

\section{Main results}

In this section we firstly review the related results, and then present the main result of this paper. For the case when r.v.s $X_{i}, 1 \leq i \leq n$, satisfy Assumption A, Bae and Ko [1] obtained the following theorem on a weighted infinite sum.

Theorem 1.A Let $\left\{X_{i}, i \geq 1\right\}$ be a sequence of nonnegative r.v.s with common distribution $F \in \mathscr{R}_{-\alpha}$, and for each $n, X_{i}, 1 \leq i \leq n$, satisfy Assumption A. If $\sum_{i=1}^{\infty}\left|\psi_{i}\right|^{p}<\infty$ for some $0<p<\min \{\alpha, 1\}$, then

$$
P\left(\sum_{i=1}^{\infty} \psi_{i} X_{i}>x\right) \sim \sum_{i \in \mathbb{I}_{+}} \bar{F}\left(\psi_{i}^{-1} x\right) \sim \bar{F}(x) \sum_{i \in \mathbb{I}_{+}} \psi_{i}^{\alpha},
$$

where $\mathbb{I}_{+}$denotes the set $\left\{i \mid \psi_{i}>0\right\}$.

For the case when r.v.s $X_{i}, 1 \leq i \leq n$, satisfy Assumption B, Geluk and Tang [12] presented a theorem as below.

Theorem 1.B Assume that $X_{i}, 1 \leq i \leq n$, are real-valued r.v.s with distributions $F_{i}$, $1 \leq i \leq n$. If $F_{i} \in \mathscr{S}$ for all $1 \leq i \leq n$ and $F_{i} * F_{j} \in \mathscr{S}$ for all $1 \leq i<j \leq n$, and Assumption B holds. Then, for all $n \geq 1$,

$$
P\left(\sum_{i=1}^{n} X_{i}>x\right) \sim \sum_{i=1}^{n} \bar{F}_{i}(x) .
$$

For the case when r.v.s $X_{i}, 1 \leq i \leq n$, satisfy Assumption $\mathrm{A}^{*}$ or $\mathrm{B}^{*}$, Jiang et al. [13] gave the following two results on sums of these r.v.s.

Theorem 1.C Assume that $X_{i}, 1 \leq i \leq n$, satisfy Assumption $\mathrm{A}^{*}$, and $F_{i} \in \mathscr{L}$ for all $1 \leq$ $i \leq n$ and $F_{i} * F_{j} \in \mathscr{S}$ for all $1 \leq i<j \leq n$. Furthermore, when these r.v.s do not satisfy Assumption $\mathrm{B}$ or $\mathrm{B}^{*}$, there exists a function $h \in \bigcap_{i=1}^{n} \mathscr{H}\left(F_{i}\right)$ such that, for all $1 \leq i \leq n$,

$$
F_{i}(-h(x))=o\left(\sum_{i=1}^{n} \overline{F_{i}}(x)\right) .
$$

Then, for all $n \geq 1$, Eq. (2.1) holds.

Theorem 1.D Assume that $X_{i}, 1 \leq i \leq n$, satisfy Assumption $\mathrm{B}^{*}$, and $F_{i} \in \mathscr{L}$ for all $1 \leq$ $i \leq n$ and $F_{i} * F_{j} \in \mathscr{S}$ for all $1 \leq i<j \leq n$. Then, for all $n \geq 1$, Eq. (2.1) holds. 
Inspired by the above results, in this paper we further consider the asymptotic tail behavior of weighted infinite sum of consistently varying tailed r.v.s under conditional dependence structure satisfying Assumption $A^{*}$ or $B^{*}$. The main results of this paper are given below.

Theorem 2.1 Let $\left\{X_{i}, i \geq 1\right\}$ be a sequence of real-valued r.v.s. with distributions $F_{i} \in \mathscr{L}$, $i \geq 1$, and all weights $\left\{\psi_{i}, i \geq 1\right\}$ be real numbers. Assume that there exists a distribution $F \in \mathscr{C}$ such that

$$
\lim \sup _{i \geq 1} \frac{F_{i}(-x)}{\bar{F}(x)}=0
$$

and

$$
0<S:=\liminf \inf _{i \geq 1} \frac{\overline{F_{i}}(x)}{\bar{F}(x)} \leq \lim \sup \sup _{i \geq 1} \frac{\overline{F_{i}}(x)}{\bar{F}(x)}=: M<\infty,
$$

and that $\sum_{i=1}^{\infty}\left|\psi_{i}\right|^{p}<\infty$ for some $0<p<\min \left\{J_{F}^{-}, J_{F}^{-} / J_{F}^{+}\right\}$, then the relation

$$
S \sum_{i \in \mathbb{I}_{+}} \bar{F}\left(\psi_{i}^{-1} x\right) \lesssim P\left(\sum_{i=1}^{\infty} \psi_{i} X_{i}>x\right) \lesssim M \sum_{i \in \mathbb{I}_{+}} \bar{F}\left(\psi_{i}^{-1} x\right)
$$

holds, if $\left\{X_{i}, i \geq 1\right\}$ is a sequence of r.v.s satisfy Assumption $\mathrm{A}^{*}$ or $\mathrm{B}^{*}$, where $\mathbb{I}_{+}$is the set given in Theorem 1.A.

Corollary 2.1 Under the conditions of Theorem 2.1, if $F_{i} \in \mathscr{C}, i \geq 1$, then

$$
S \sum_{i \in \mathbb{I}_{+}} \bar{F}\left(\psi_{i}^{-1} x\right) \lesssim P\left(\sum_{i=1}^{\infty} \psi_{i} X_{i}>x\right) \sim \sum_{i \in \mathbb{I}_{+}} \bar{F}_{i}\left(\psi_{i}^{-1} x\right) \lesssim M \sum_{i \in \mathbb{I}_{+}} \bar{F}\left(\psi_{i}^{-1} x\right)
$$

and furthermore if $F_{i} \sim F, i \geq 1$, then

$$
P\left(\sum_{i=1}^{\infty} \psi_{i} X_{i}>x\right) \sim \sum_{i \in \mathbb{I}_{+}} \bar{F}\left(\psi_{i}^{-1} x\right)
$$

If $F_{i} \sim F \in \mathscr{R}_{-\alpha}, i \geq 1$, then

$$
P\left(\sum_{i=1}^{\infty} \psi_{i} X_{i}>x\right) \sim \sum_{i \in \mathbb{I}_{+}} \bar{F}\left(\psi_{i}^{-1} x\right) \sim \bar{F}(x) \sum_{i \in \mathbb{I}_{+}} \psi_{i}^{\alpha}
$$

\section{Lemmas}

In order to prove Theorem 2.1 and Corollary 2.1, we now give two lemmas which are concerned with the case that weights $\left\{\psi_{i}, i \geq 1\right\}$ be positive.

Lemma 3.1 Let $\left\{X_{i}, i \geq 1\right\}$ be a sequence of real-valued r.v.s with their respective distributions $F_{i} \in \mathscr{L}, i \geq 1$, and their weights $\left\{\psi_{i}, i \geq 1\right\}$ be positive. Assume that there exists a distribution $F \in \mathscr{C}$ such that (2.2) and (2.3) hold, and that $\sum_{i=1}^{\infty} \psi_{i}^{p}<\infty$ for some 
$0<p<\min \left\{J_{F}^{-}, J_{F}^{-} / J_{F}^{+}\right\}$, then the relation

$$
S \sum_{i=1}^{\infty} \bar{F}\left(\psi_{i}^{-1} x\right) \lesssim P\left(\sum_{i=1}^{\infty} \psi_{i} X_{i}>x\right) \lesssim M \sum_{i=1}^{\infty} \bar{F}\left(\psi_{i}^{-1} x\right)
$$

holds, if $\left\{X_{i}, i \geq 1\right\}$ is a sequence of r.v.s satisfy Assumption $\mathrm{A}^{*}$ or $\mathrm{B}^{*}$.

Proof Without loss of generality, we assume that $0<\psi_{i} \leq 1, i \geq 1$. It is because there can be only a finite number of terms with $\psi_{i}>1$ by the assumption and, if that is the case, we can divide each weight with the maximum of such $\psi_{i}$ s.

Take $0<p<\min \left\{J_{F}^{-}, J_{F}^{-} / J_{F}^{+}\right\}$such that $\sum_{i=1}^{\infty} \psi_{i}^{p}<\infty$. Then, for any $0<\varepsilon<1$, there exists a large positive integer $n_{0}$ such that

$$
\sum_{i=n_{0}+1}^{\infty} \psi_{i}^{p}<\varepsilon
$$

For the above integer $n_{0}$, by $F \in \mathscr{C} \subset \mathscr{D},(1.1)$ and (3.2), there exist positive constants $C_{3}$ and $D_{3}$ such that, for all large $x \geq D_{3}$ and the above $p$,

$$
\sum_{i=n_{0}+1}^{\infty} \bar{F}\left(\psi_{i}^{-1} x\right) \leq C_{3} \bar{F}(x) \sum_{i=n_{0}+1}^{\infty} \psi_{i}^{p} \leq C_{3} \varepsilon \bar{F}(x) .
$$

Firstly, to prove the upper bound of Eq. (3.1), we follow the approach used in the proof of Lemma 4.24 in Resnick [19] or Theorem 2 in Bae and Ko [1]. For any $0<\delta<1$ and integer $n_{0}$ in (3.2), we have

$$
\begin{aligned}
P\left(\sum_{i=1}^{\infty} \psi_{i} X_{i}>x\right) & \leq P\left(\sum_{i=1}^{n_{0}} \psi_{i} X_{i}^{+}>(1-\delta) x\right)+P\left(\sum_{i=n_{0}+1}^{\infty} \psi_{i} X_{i}^{+}>\delta x\right) \\
& =: I_{1}(x)+I_{2}(x),
\end{aligned}
$$

where $X_{i}^{+}=\max \left\{X_{i}, 0\right\}, i \geq 1$. For convenience's sake, we remark that $F_{i} \in \mathscr{L} \cap \mathscr{D}, i \geq 1$, can imply $F_{i} \in \mathscr{S}, 1 \leq i \leq n$, and $F_{i} * F_{j} \in \mathscr{S}$ for all $1 \leq i<j \leq n$; see Jiang et al. [13]. Therefore, the distributions $F_{i}, i \geq 1$, in Theorem 2.1 and Lemma 3.1, can also satisfy the conditions in Theorem 1.C. For $I_{1}(x)$, by Theorem 1.C or 1.D, and (2.3), it follows that

$$
\begin{aligned}
I_{1}(x) & \sim \sum_{i=1}^{n_{0}} P\left(\psi_{i} X_{i}^{+}>(1-\delta) x\right) \\
& \lesssim M \sum_{i=1}^{n_{0}} \bar{F}\left(\psi_{i}^{-1}(1-\delta) x\right) \\
& \leq M \sup _{0<\psi_{i} \leq 1} \frac{\bar{F}\left(\psi_{i}^{-1}(1-\delta) x\right)}{\bar{F}\left(\psi_{i}^{-1} x\right)} \sum_{i=1}^{n_{0}} \bar{F}\left(\psi_{i}^{-1} x\right) .
\end{aligned}
$$

By $F \in \mathscr{C}$, we get

$$
\lim _{\delta \downarrow 0} \limsup _{x \rightarrow \infty} \sup _{0<\psi_{i} \leq 1} \frac{\bar{F}\left(\psi_{i}^{-1}(1-\delta) x\right)}{\bar{F}\left(\psi_{i}^{-1} x\right)}=\lim _{\delta \downarrow 0} \limsup _{x \rightarrow \infty} \frac{\bar{F}((1-\delta) x)}{\bar{F}(x)}=1 .
$$


Hence, we substitute (3.6) into (3.5) to obtain

$$
I_{1}(x) \lesssim M \sum_{i=1}^{n_{0}} \bar{F}\left(\psi_{i}^{-1} x\right)
$$

For $I_{2}(x)$, when $0<J_{F}^{+}<1$, we have

$$
\begin{aligned}
I_{2}(x) & \leq P\left(\bigcup_{i=n_{0}+1}^{\infty}\left\{\psi_{i} X_{i}^{+}>\delta x\right\}\right)+P\left(\sum_{i=n_{0}+1}^{\infty} \psi_{i} X_{i}^{+} \mathbf{1}_{\left\{\psi_{i} X_{i}^{+} \leq \delta x\right\}}>\delta x\right) \\
& =: I_{21}(x)+I_{22}(x) .
\end{aligned}
$$

By (1.2), (2.3), (3.3) and $F \in \mathscr{C} \subset \mathscr{D}$, for any $p_{2}>J_{F}^{+}$, there exist some large positive constants $C_{4}$ and $D_{4}$ such that, for all $x \geq \max \left\{D_{3}, D_{4}\right\}$,

$$
\begin{aligned}
I_{21}(x) & \leq \sum_{i=n_{0}+1}^{\infty} P\left(\psi_{i} X_{i}^{+}>\delta x\right) \\
& \lesssim M \sum_{i=n_{0}+1}^{\infty} \bar{F}\left(\psi_{i}^{-1} \delta x\right) \\
& \leq C_{3} C_{4} M \delta^{-p_{2}} \varepsilon \bar{F}(x) .
\end{aligned}
$$

By Markov's inequality and the monotone convergence theorem, we see that

$$
\begin{aligned}
I_{22}(x) & \leq(\delta x)^{-1} E\left(\sum_{i=n_{0}+1}^{\infty} \psi_{i} X_{i}^{+} \mathbf{1}_{\left\{\psi_{i} X_{i}^{+} \leq \delta x\right\}}\right) \\
& =(\delta x)^{-1} \sum_{i=n_{0}+1}^{\infty} \psi_{i} E\left(X_{i}^{+} \mathbf{1}_{\left\{X_{i}^{+} \leq \psi_{i}^{-1} \delta x\right\}}\right) .
\end{aligned}
$$

By $F \in \mathscr{C} \subset \mathscr{D},(1.2)$ and (2.3), for any $J_{F}^{+}<p_{2}<1$, there exist some large positive constants $C_{5}$ and $D_{5}$ such that, for all $x \geq D_{5}$,

$$
\begin{aligned}
E\left(X_{i}^{+} \mathbf{1}_{\left\{X_{i}^{+} \leq \psi_{i}^{-1} \delta x\right\}}\right) & =-\int_{0}^{\psi_{i}^{-1} \delta x} u d \overline{F_{i}}(u) \\
& =-\psi_{i}^{-1} \delta x \overline{F_{i}}\left(\psi_{i}^{-1} \delta x\right)+\int_{0}^{\psi_{i}^{-1} \delta x} \overline{F_{i}}(u) d u \\
& \leq \psi_{i}^{-1} \delta x \int_{0}^{1} \overline{F_{i}}\left(t \psi_{i}^{-1} \delta x\right) d t \\
& \lesssim M \psi_{i}^{-1} \delta x \bar{F}\left(\psi_{i}^{-1} \delta x\right) \int_{0}^{1} \frac{\bar{F}\left(t \psi_{i}^{-1} \delta x\right)}{\bar{F}\left(\psi_{i}^{-1} \delta x\right)} d t \\
& \leq \frac{C_{5} M}{1-p_{2}} \psi_{i}^{-1} \delta x \bar{F}\left(\psi_{i}^{-1} \delta x\right)
\end{aligned}
$$

Substituting (3.11) into (3.10) and using the last step of (3.9) can lead to

$$
I_{22}(x) \lesssim \frac{C_{3} C_{4} C_{5} M}{1-p_{2}} \delta^{-p_{2}} \varepsilon \bar{F}(x)
$$


Therefore by (3.4), (3.7)-(3.9), (3.12) and the arbitrariness of $\varepsilon$, we derive that

$$
P\left(\sum_{i=1}^{\infty} \psi_{i} X_{i}>x\right) \lesssim M \sum_{i=1}^{n_{0}} \bar{F}\left(\psi_{i}^{-1} x\right) \leq M \sum_{i=1}^{\infty} \bar{F}\left(\psi_{i}^{-1} x\right)
$$

For the case when $J_{F}^{+} \geq 1$, we choose some constant $\beta \in\left(J_{F}^{+}, J_{F}^{-} p^{-1}\right)$ such that $p<\beta^{-1} J_{F}^{-} \leq$ $\beta^{-1} J_{F}^{+}<1$. Set $\psi=\sum_{i=n_{0}+1}^{\infty} \psi_{i}$, which is assumed to be less than 1 without loss of generality. Then by Jensen's inequality, it follows that

$$
\begin{aligned}
I_{2}(x)= & P\left(\psi^{\beta}\left(\sum_{i=n_{0}+1}^{\infty} \frac{\psi_{i}}{\psi} X_{i}^{+}\right)^{\beta}>\delta^{\beta} x^{\beta}\right) \\
\leq & P\left(\sum_{i=n_{0}+1}^{\infty} \psi_{i} X_{i}^{+\beta}>\psi^{1-\beta} \delta^{\beta} x^{\beta}\right) \\
\leq & P\left(\bigcup_{i=n_{0}+1}^{\infty}\left\{\psi_{i} X_{i}^{+\beta}>\psi^{1-\beta} \delta^{\beta} x^{\beta}\right\}\right) \\
& +P\left(\sum_{i=n_{0}+1}^{\infty} \psi_{i} X_{i}^{+\beta} \mathbf{1}_{\left\{\psi_{i} X_{i}^{+\beta} \leq \psi^{1-\beta} \delta^{\beta} x^{\beta}\right\}}>\psi^{1-\beta} \delta^{\beta} x^{\beta}\right) \\
= & I_{21}^{\prime}(x)+I_{22}^{\prime}(x) .
\end{aligned}
$$

For $I_{21}^{\prime}(x)$, by using $F \in \mathscr{C} \subset \mathscr{D}$ and (1.1), and arguing as (3.9), for any $p_{1} \in\left(\beta p, J_{F}^{-}\right)$ and $p_{2}>J_{F}^{+}$, there exist some large positive constants $C_{6}$ and $D_{6}$ such that, for all $x \geq$ $\max \left\{D_{3}, D_{4}, D_{6}\right\}$,

$$
\begin{aligned}
I_{21}^{\prime}(x) & \leq \sum_{i=n_{0}+1}^{\infty} P\left(X_{i}^{+}>\psi_{i}^{-\frac{1}{\beta}} \psi^{\frac{1-\beta}{\beta}} \delta x\right) \\
& \lesssim M \sum_{i=n_{0}+1}^{\infty} \bar{F}\left(\psi_{i}^{-\frac{1}{\beta}} \psi^{\frac{1-\beta}{\beta}} \delta x\right) \\
& \leq C_{6} M \psi \frac{p_{1}(\beta-1)}{\beta} \sum_{i=n_{0}+1}^{\infty} \bar{F}\left(\psi_{i}^{-\frac{1}{\beta}} \delta x\right) \\
& \leq C_{3} C_{4} C_{6} M \psi^{\frac{p_{1}(\beta-1)}{\beta}} \delta^{-p_{2}} \varepsilon \bar{F}(x) .
\end{aligned}
$$

For $I_{22}^{\prime}(x)$, by going along the same lines of the derivation of $I_{22}(x)$, we conclude that, for any $J_{F}^{+}<p_{2}<\beta$, there exist some large positive constants $C_{7}$ and $D_{7}$ such that, for all $x \geq$ $\max \left\{D_{3}, D_{4}, D_{6}, D_{7}\right\}$,

$$
\begin{aligned}
I_{22}^{\prime}(x) & \lesssim \frac{C_{7} M \beta}{\beta-p_{2}} \sum_{i=n_{0}+1}^{\infty} \bar{F}\left(\psi_{i}^{-\beta} \psi^{\frac{1-\beta}{\beta}} \delta x\right) \\
& \leq \frac{C_{3} C_{4} C_{6} C_{7} M \beta}{\beta-p_{2}} \psi^{\frac{p_{1}(\beta-1)}{\beta}} \delta^{-p_{2}} \varepsilon \bar{F}(x),
\end{aligned}
$$

where the last step is obtained similarly to (3.15). Then by (3.4), (3.7), (3.14)-(3.16) and the arbitrariness of $\varepsilon$, we prove that Eq. (3.13) holds. 
Secondly, we deal with the lower bound of Eq. (3.1). Let $n_{0}$ and $p$ be fixed as those in (3.2). For any $0<\delta<1$, we have

$$
\begin{aligned}
P\left(\sum_{i=1}^{\infty} \psi_{i} X_{i}>x\right) & =P\left(\sum_{i=1}^{\infty} \psi_{i} X_{i}^{+}-\sum_{i=1}^{\infty} \psi_{i} X_{i}^{-}>x\right) \\
& \geq P\left(\sum_{i=1}^{\infty} \psi_{i} X_{i}^{+}>(1+\delta) x, \sum_{i=1}^{\infty} \psi_{i} X_{i}^{-} \leq \delta x\right) \\
& \geq P\left(\sum_{i=1}^{\infty} \psi_{i} X_{i}^{+}>(1+\delta) x\right)-P\left(\sum_{i=1}^{\infty} \psi_{i} X_{i}^{-}>\delta x\right) \\
& =: I_{3}(x)-I_{4}(x),
\end{aligned}
$$

where $X_{i}^{-}=-\min \left\{X_{i}, 0\right\}, i \geq 1$. For $I_{3}(x)$, by (2.3), Theorem 1.C or 1.D, we have

$$
\begin{aligned}
I_{3}(x) & \geq P\left(\sum_{i=1}^{n_{0}} \psi_{i} X_{i}^{+}>(1+\delta) x\right) \\
& \sim \sum_{i=1}^{n_{0}} P\left(\psi_{i} X_{i}^{+}>(1+\delta) x\right) \\
& \gtrsim S \sum_{i=1}^{n_{0}} \bar{F}\left(\psi_{i}^{-1}(1+\delta) x\right) \\
& \geq S \inf _{0<\psi_{i} \leq 1} \frac{\bar{F}\left(\psi_{i}^{-1}(1+\delta) x\right)}{\bar{F}\left(\psi_{i}^{-1} x\right)} \sum_{i=1}^{n_{0}} \bar{F}\left(\psi_{i}^{-1} x\right) .
\end{aligned}
$$

By $F \in \mathscr{C}$, it follows that

$$
\lim _{\delta \downarrow 0} \liminf _{x \rightarrow \infty} \inf _{0<\psi_{i} \leq 1} \frac{\bar{F}\left(\psi_{i}^{-1}(1+\delta) x\right)}{\bar{F}\left(\psi_{i}^{-1} x\right)}=\lim _{\delta \downarrow 0} \liminf _{x \rightarrow \infty} \frac{\bar{F}((1+\delta) x)}{\bar{F}(x)}=1 .
$$

By (3.3), (3.18) and (3.19), we obtain

$$
\begin{aligned}
I_{3}(x) & \gtrsim S \sum_{i=1}^{n_{0}} \bar{F}\left(\psi_{i}^{-1} x\right) \\
& =S \sum_{i=1}^{\infty} \bar{F}\left(\psi_{i}^{-1} x\right)-S \sum_{i=n_{0}+1}^{\infty} \bar{F}\left(\psi_{i}^{-1} x\right) \\
& \geq S \sum_{i=1}^{\infty} \bar{F}\left(\psi_{i}^{-1} x\right)-C_{3} S \varepsilon \bar{F}(x)
\end{aligned}
$$

which, along with the arbitrariness of $0<\varepsilon<1$, implies that

$$
I_{3}(x) \gtrsim S \sum_{i=1}^{\infty} \bar{F}\left(\psi_{i}^{-1} x\right)
$$


By (2.2), for any $0<\varepsilon<1$, there exists a large positive constant $D^{\prime}$ such that, for all $x \geq D^{\prime}$,

$$
\sup _{i \geq 1} \frac{F_{i}(-x)}{\bar{F}(x)}<\varepsilon
$$

For $I_{4}(x)$, we only consider the case $0<J_{F}^{+}<1$. In fact, the case of $J_{F}^{+} \geq 1$ follows from similar derivations to (3.14)-(3.16) with slight modifications. Clearly,

$$
\begin{aligned}
I_{4}(x) & \leq \sum_{i=1}^{\infty} P\left(\psi_{i} X_{i}^{-}>\delta x\right)+P\left(\sum_{i=1}^{\infty} \psi_{i} X_{i}^{-} \mathbf{1}_{\left\{\psi_{i} X_{i}^{-} \leq \delta x\right\}}>\delta x\right) \\
& =\sum_{i=1}^{\infty} P\left(\psi_{i} X_{i}<-\delta x\right)+P\left(\sum_{i=1}^{\infty} \psi_{i} X_{i}^{-} \mathbf{1}_{\left\{\psi_{i} X_{i}^{-} \leq \delta x\right\}}>\delta x\right) \\
& =: I_{41}(x)+I_{42}(x) .
\end{aligned}
$$

For $I_{41}(x)$, by (3.21) and the last step of (3.9), for all $x \geq \max \left\{D^{\prime}, D_{4}\right\}$,

$$
I_{41}(x)<\varepsilon \sum_{i=1}^{\infty} \bar{F}\left(\psi_{i}^{-1} \delta x\right) \leq C_{4} \delta^{-p_{2}} \varepsilon \sum_{i=1}^{\infty} \bar{F}\left(\psi_{i}^{-1} x\right) .
$$

For $I_{42}(x)$, similarly to (3.10), we have

$$
I_{42}(x) \leq(\delta x)^{-1} \sum_{i=1}^{\infty} \psi_{i} E\left(X_{i}^{-} \mathbf{1}_{\left\{X_{i}^{-} \leq \psi_{i}^{-1} \delta x\right\}}\right)
$$

Similarly to (3.11), by $F \in \mathscr{C} \subset \mathscr{D},(1.2)$, (2.2) and (2.3), for any $J_{F}^{+}<p_{2}<1$, there exist some large positive constants $C_{8}$ and $D_{8}$ such that, for all $x \geq \max \left\{D^{\prime}, D_{8}\right\}$,

$$
\begin{aligned}
& E\left(X_{i}^{-} \mathbf{1}_{\left\{X_{i}^{-} \leq \psi_{i}^{-1} \delta x\right\}}\right) \\
& \quad=-\psi_{i}^{-1} \delta x P\left(X_{i}^{-}>\psi_{i}^{-1} \delta x\right)+\psi_{i}^{-1} \delta x \int_{0}^{1} P\left(X_{i}^{-}>t \psi_{i}^{-1} \delta x\right) d t \\
& \quad \leq \psi_{i}^{-1} \delta x \bar{F}\left(\psi_{i}^{-1} \delta x\right) \int_{0}^{1} \frac{F_{i}\left(-t \psi_{i}^{-1} \delta x\right)}{\bar{F}\left(t \psi_{i}^{-1} \delta x\right)} \frac{\bar{F}\left(t \psi_{i}^{-1} \delta x\right)}{\bar{F}\left(\psi_{i}^{-1} \delta x\right)} d t \\
& \quad<\varepsilon \psi_{i}^{-1} \delta x \bar{F}\left(\psi_{i}^{-1} \delta x\right) \int_{0}^{1} \frac{\bar{F}\left(t \psi_{i}^{-1} \delta x\right)}{\bar{F}\left(\psi_{i}^{-1} \delta x\right)} d t \\
& \quad \leq \frac{C_{8} \varepsilon}{1-p_{2}} \psi_{i}^{-1} \delta x \bar{F}\left(\psi_{i}^{-1} \delta x\right) .
\end{aligned}
$$

Then, by substituting (3.25) into (3.24) and arguing similarly to (3.9), we prove that, for all $x \geq \max \left\{D^{\prime}, D_{4}, D_{8}\right\}$,

$$
I_{42}(x)<\frac{C_{8} \varepsilon}{1-p_{2}} \sum_{i=1}^{\infty} \bar{F}\left(\psi_{i}^{-1} \delta x\right) \leq \frac{C_{4} C_{8}}{1-p_{2}} \delta^{-p_{2}} \varepsilon \sum_{i=1}^{\infty} \bar{F}\left(\psi_{i}^{-1} x\right)
$$

and further we substitute (3.23) and (3.26) into (3.22) to obtain, for all $x \geq \max \left\{D^{\prime}, D_{4}, D_{8}\right\}$,

$$
I_{4}(x)<\left(\frac{C_{8}}{1-p_{2}}+1\right) C_{4} \delta^{-p_{2}} \varepsilon \sum_{i=1}^{\infty} \bar{F}\left(\psi_{i}^{-1} x\right)
$$


which, along with (3.17), (3.20) and the arbitrariness of $0<\varepsilon<1$, can show the lower bound of Eq. (3.1).

Lemma 3.2 Under the conditions of Lemma 3.1, if $F_{i} \in \mathscr{C}, i \geq 1$, then

$$
S \sum_{i=1}^{\infty} \bar{F}\left(\psi_{i}^{-1} x\right) \lesssim P\left(\sum_{i=1}^{\infty} \psi_{i} X_{i}>x\right) \sim \sum_{i=1}^{\infty} \bar{F}_{i}\left(\psi_{i}^{-1} x\right) \lesssim M \sum_{i=1}^{\infty} \bar{F}\left(\psi_{i}^{-1} x\right),
$$

and further if $F_{i} \sim F, i \geq 1$, then

$$
P\left(\sum_{i=1}^{\infty} \psi_{i} X_{i}>x\right) \sim \sum_{i=1}^{\infty} \bar{F}\left(\psi_{i}^{-1} x\right)
$$

If $F_{i} \sim F \in \mathscr{R}_{-\alpha}, i \geq 1$, then

$$
P\left(\sum_{i=1}^{\infty} \psi_{i} X_{i}>x\right) \sim \sum_{i=1}^{\infty} \bar{F}\left(\psi_{i}^{-1} x\right) \sim \bar{F}(x) \sum_{i=1}^{\infty} \psi_{i}^{\alpha} .
$$

Proof By Lemma 3.1, it suffices to prove that

$$
P\left(\sum_{i=1}^{\infty} \psi_{i} X_{i}>x\right) \sim \sum_{i=1}^{\infty} \overline{F_{i}}\left(\psi_{i}^{-1} x\right)
$$

and, when $F \in \mathscr{R}_{-\alpha}$,

$$
\sum_{i=1}^{\infty} \bar{F}\left(\psi_{i}^{-1} x\right) \sim \bar{F}(x) \sum_{i=1}^{\infty} \psi_{i}^{\alpha}
$$

Firstly, we prove (3.28). By the proof of Lemma 3.1, we only need to prove

$$
I_{1}(x) \lesssim \sum_{i=1}^{\infty} \overline{F_{i}}\left(\psi_{i}^{-1} x\right)
$$

and

$$
I_{3}(x) \gtrsim \sum_{i=1}^{\infty} \overline{F_{i}}\left(\psi_{i}^{-1} x\right)
$$

Since $F_{i} \in \mathscr{C}, i \geq 1$, we know that

$$
\lim _{\delta \downarrow 0} \limsup _{x \rightarrow \infty} \frac{\overline{F_{i}}\left(\psi_{i}^{-1}(1-\delta) x\right)}{\overline{F_{i}}\left(\psi_{i}^{-1} x\right)}=\lim _{\delta \downarrow 0} \limsup _{x \rightarrow \infty} \frac{\overline{F_{i}}((1-\delta) x)}{\overline{F_{i}}(x)}=1
$$

and

$$
\lim _{\delta \downarrow 0} \liminf _{x \rightarrow \infty} \frac{\overline{F_{i}}\left(\psi_{i}^{-1}(1+\delta) x\right)}{\bar{F}_{i}\left(\psi_{i}^{-1} x\right)}=\lim _{\delta \downarrow 0} \liminf _{x \rightarrow \infty} \frac{\overline{F_{i}}((1+\delta) x)}{\bar{F}_{i}(x)}=1 .
$$


By (3.32) and Theorem 1.C or 1.D, it follows that

$$
I_{1}(x) \sim \sum_{i=1}^{n_{0}} \overline{F_{i}}\left(\psi_{i}^{-1}(1-\delta) x\right) \sim \sum_{i=1}^{n_{0}} \overline{F_{i}}\left(\psi_{i}^{-1} x\right) \leq \sum_{i=1}^{\infty} \overline{F_{i}}\left(\psi_{i}^{-1} x\right)
$$

which leads to (3.30). By Theorem 1.C or 1.D, (2.3), (3.3) and (3.33), we have

$$
\begin{aligned}
I_{3}(x) & \gtrsim \sum_{i=1}^{n_{0}} \overline{F_{i}}\left(\psi_{i}^{-1}(1+\delta) x\right) \sim \sum_{i=1}^{n_{0}} \overline{F_{i}}\left(\psi_{i}^{-1} x\right) \\
& \gtrsim \sum_{i=1}^{\infty} \overline{F_{i}}\left(\psi_{i}^{-1} x\right)-M \sum_{i=n_{0}+1}^{\infty} \bar{F}\left(\psi_{i}^{-1} x\right) \\
& \geq \sum_{i=1}^{\infty} \overline{F_{i}}\left(\psi_{i}^{-1} x\right)-C_{3} M \varepsilon \bar{F}(x)
\end{aligned}
$$

which, along with the arbitrariness of $0<\varepsilon<1$, implies that Eq. (3.31) holds.

Secondly, we prove (3.29). By $F \in \mathscr{R}_{-\alpha}$ and the control convergence theorem, we have

$$
\sum_{i=1}^{\infty} \bar{F}\left(\psi_{i}^{-1} x\right)=\bar{F}(x) \sum_{i=1}^{\infty} \frac{\bar{F}\left(\psi_{i}^{-1} x\right)}{\bar{F}(x)} \sim \bar{F}(x) \sum_{i=1}^{\infty} \psi_{i}^{\alpha}
$$

\section{Proof of main result}

In this section, we will prove the main result of this paper.

Proof of Theorem 2.1 Without loss of generality, we may assume that $-1 \leq \psi_{i} \leq 1$. Firstly, we consider the upper bound of $\mathrm{E}(2.4)$. For any $0<\delta<1$, we have

$$
\begin{aligned}
P\left(\sum_{i=1}^{\infty} \psi_{i} X_{i}>x\right) & =P\left(\sum_{i=\mathbb{I}_{+}} \psi_{i} X_{i}+\sum_{i=\mathbb{I}_{-}} \psi_{i} X_{i}>x\right) \\
& \leq P\left(\sum_{i \in \mathbb{I}_{+}} \psi_{i} X_{i}>(1-\delta) x\right)+P\left(\sum_{i \in \mathbb{I}_{-}} \psi_{i} X_{i}>\delta x\right) \\
& =: I_{5}(x)+I_{6}(x),
\end{aligned}
$$

where $\mathbb{I}_{-}$denotes the set $\left\{i \mid \psi_{i}<0\right\}$. For $I_{5}(x)$, by Lemma 3.1 and (3.6), we have

$$
\begin{aligned}
I_{5}(x) & \lesssim M \sum_{i \in \mathbb{I}_{+}} \bar{F}\left(\psi_{i}^{-1}(1-\delta) x\right) \\
& \leq M \sup _{0<\psi_{i} \leq 1} \frac{\bar{F}\left(\psi_{i}^{-1}(1-\delta) x\right)}{\bar{F}\left(\psi_{i}^{-1} x\right)} \sum_{i \in \mathbb{I}_{+}} \bar{F}\left(\psi_{i}^{-1} x\right) \\
& \sim M \sum_{i \in \mathbb{I}_{+}} \bar{F}\left(\psi_{i}^{-1} x\right) .
\end{aligned}
$$


For $I_{6}(x)$, it follows from (3.27) that, for all $x \geq \max \left\{D^{\prime}, D_{4}, D_{8}\right\}$,

$$
I_{6}(x) \leq P\left(\sum_{i \in \mathbb{I}_{-}}\left|\psi_{i}\right| X_{i}^{-}>\delta x\right)<\left(\frac{C_{8}}{1-p_{2}}+1\right) C_{4} \delta^{-p_{2}} \varepsilon \sum_{i=1}^{\infty} \bar{F}\left(\left|\psi_{i}\right|^{-1} x\right) .
$$

Thus, substituting (4.2) and (4.3) into (4.1) and considering the arbitrariness of $0<\varepsilon<1$, we show that

$$
P\left(\sum_{i=1}^{\infty} \psi_{i} X_{i}>x\right) \lesssim M \sum_{i \in \mathbb{I}_{+}} \bar{F}\left(\psi_{i}^{-1} x\right)
$$

Secondly, we consider the lower bound of Eq. (2.4). By Lemma 3.1 and (3.19), we derive that

$$
\begin{aligned}
P\left(\sum_{i=1}^{\infty} \psi_{i} X_{i}>x\right) & =P\left(\sum_{i \in \mathbb{I}_{+}} \psi_{i} X_{i}+\sum_{i \in \mathbb{I}_{-}} \psi_{i} X_{i}>x\right) \\
& \geq P\left(\sum_{i \in \mathbb{I}_{+}} \psi_{i} X_{i}>(1+\delta) x, \sum_{i \in \mathbb{I}_{-}} \psi_{i} X_{i} \geq-\delta x\right) \\
& \sim P\left(\sum_{i \in \mathbb{I}_{+}} \psi_{i} X_{i}>(1+\delta) x\right) \\
& \sim \sum_{i \in \mathbb{I}_{+}} P\left(\psi_{i} X_{i}>(1+\delta) x\right) \\
& \geq S \sum_{i \in \mathbb{I}_{+}} \bar{F}\left(\psi_{i}^{-1}(1+\delta) x\right) \\
& \geq S \inf _{0<\psi_{i}<1} \frac{\bar{F}\left(\psi_{i}^{-1}(1+\delta) x\right)}{\bar{F}\left(\psi_{i}^{-1} x\right)} \sum_{i \in \mathbb{I}_{+}} \bar{F}\left(\psi_{i}^{-1} x\right) \\
& \sim S \sum_{i \in \mathbb{I}_{+}} \bar{F}\left(\psi_{i}^{-1} x\right),
\end{aligned}
$$

where in the third step we used the fact that the event $\left\{\omega: \sum_{i \in \mathbb{I}_{-}} \psi_{i} X_{i} \geq-\delta x\right\}$ increases to a certain event as $x$ tends to infinity. Therefore, we combine (4.4) and (4.5) to conclude that Eq. (2.4) holds.

Proof of Corollary 2.1 By (3.29) and the proof of Theorem 2.1, we only need to prove

$$
I_{5}(x) \lesssim \sum_{i \in \mathbb{I}_{+}} \bar{F}_{i}\left(\psi_{i}^{-1} x\right)
$$

and

$$
P\left(\sum_{i=1}^{\infty} \psi_{i} X_{i}>x\right) \gtrsim \sum_{i \in \mathbb{I}_{+}} \overline{F_{i}}\left(\psi_{i}^{-1} x\right)
$$


Firstly, we consider (4.6). By Lemma 3.2, (3.3) and (3.32), we conclude that, for any $p_{2}>$ $J_{F}^{+}$, there exist some large positive constants $C_{9}$ and $D_{9}$ such that, for all $x \geq \max \left\{D_{3}, D_{9}\right\}$,

$$
\begin{aligned}
I_{5}(x) & \sim \sum_{i \in \mathbb{I}_{+}} \bar{F}_{i}\left(\psi_{i}^{-1}(1-\delta) x\right) \\
& \lesssim \sum_{i \in \mathbb{I}_{+}, i \leq n_{0}} \bar{F}_{i}\left(\psi_{i}^{-1}(1-\delta) x\right)+M \sum_{i \in \mathbb{I}_{+}, i \geq n_{0}+1} \bar{F}\left(\psi_{i}^{-1}(1-\delta) x\right) \\
& \lesssim \sum_{i \in \mathbb{I}_{+}, i \leq n_{0}} \bar{F}_{i}\left(\psi_{i}^{-1} x\right)+C_{3} C_{9} M(1-\delta)^{-p_{2}} \varepsilon \bar{F}(x),
\end{aligned}
$$

which, along with the arbitrariness of $0<\varepsilon<1$, proves (4.6).

Secondly, we consider (4.7). Similarly to (4.5), by Theorem 3.1, (3.3) and (3.33), we conclude that

$$
\begin{aligned}
P\left(\sum_{i=1}^{\infty} \psi_{i} X_{i}>x\right) & \gtrsim \sum_{i \in \mathbb{I}_{+}} \bar{F}_{i}\left(\psi_{i}^{-1}(1+\delta) x\right) \\
& \geq \sum_{i \in \mathbb{I}_{+}, i \leq n_{0}} \bar{F}_{i}\left(\psi_{i}^{-1}(1+\delta) x\right) \\
& \sim \sum_{i \in \mathbb{I}_{+}, i \leq n_{0}} \bar{F}_{i}\left(\psi_{i}^{-1} x\right) \\
& \gtrsim \sum_{i \in \mathbb{I}_{+}} \bar{F}\left(\psi_{i}^{-1} x\right)-M \sum_{i \in \mathbb{I}_{+}, i \geq n_{0}+1} \bar{F}\left(\psi_{i}^{-1} x\right) \\
& \geq \sum_{i \in \mathbb{I}_{+}} \bar{F}\left(\psi_{i}^{-1} x\right)-C_{3} M \varepsilon \bar{F}(x),
\end{aligned}
$$

which, along with the arbitrariness of $0<\varepsilon<1$, proves (4.7).

\section{Acknowledgements}

The authors would like to thank the two referees and the editors for their very valuable comments on an earlier version of the paper.

\section{Funding}

This research was supported by the National Natural Science Foundation of China (Nos. 11501295 and 11871289), the Postdoctoral Science Foundation of China (No. 2015M580415), the Natural Science Foundation of Jiangsu Province of China (No. BK20151459), the Social Science Foundation of Jiangsu Province of China (No. 16GLC006), the Postdoctoral Science Foundation of Jiangsu Province of China (No. 1501004B) and Qing-Lan Project of Jiangsu Province.

Availability of data and materials

No data were used to support this study.

Competing interests

The authors declare that they have no competing interests.

\section{Authors' contributions}

The author QG found the main reference Bae and Ko [1] in the literature study, and proposed the ideas and methods of the main results in our paper. All authors implemented concretely the above ideas and methods, and accomplished this paper. All the authors read and approved the final manuscript.

\section{Author details}

${ }^{1}$ School of Statistics and Mathematics, Nanjing Audit University, Nanjing, China. ${ }^{2}$ Department of Industrial and Systems Engineering, University at Buffalo, Buffalo, USA. ${ }^{3}$ Aviation Maintenance NCO Academy, Air Force Engineering University, Xinyang, China.

\section{Publisher's Note}

Springer Nature remains neutral with regard to jurisdictional claims in published maps and institutional affiliations. 
Received: 27 August 2018 Accepted: 14 April 2019 Published online: 03 May 2019

\section{References}

1. Bae, T., Ko, B.: A note on weighted infinite sums of dependent regularly varying tailed random variables. J. Korean Stat. Soc. 46, 321-327 (2017)

2. Bingham, N.H., Goldie, C.M., Teugels, J.M.: Regular Variation. Cambridge University Press, Cambridge (1987)

3. Chen, Y., Wang, L., Wang, Y.: Uniform asymptotics for the finite-time ruin probabilities of two kinds of nonstandard bidimensional risk models. J. Math. Anal. Appl. 401, 114-129 (2013)

4. Chen, Y., Yuan, K.C.: Sums of pairwise quasi-asymptotically independent random variables with consistent variation. Stoch. Models 25(1), 76-89 (2009)

5. Embrechts, P., Klüppelberg, C., Mikosch, T.: Modelling Extremal Events for Insurance and Finance. Springer, Berlin (1997)

6. Foss, S., Richards, A.: On sums of conditionally independent subexponential random variables. Math. Oper. Res. 35 102-119 (2010)

7. Gao, M., Wang, K., Chen, L.: Precise large deviations for widely orthant dependent random variables with different distributions. J. Inequal. Appl. (2018). https://doi.org/10.1186/s13660-018-1613-2

8. Gao, Q., Jin, N.: Randomly weighted sums of pairwise quasi upper-tail independent increments with application to risk theory. Commun. Stat., Theory Methods 44(18), 3885-3902 (2015)

9. Gao, Q., Liu, X.: Uniform asymptotics for the finite-time ruin probability with upper tail asymptotically independent claims and constant force of interest. Stat. Probab. Lett. 83, 1527-1538 (2013)

10. Gao, Q., Wang, Y.: Randomly weighted sums with dominated varying-tailed increments and application to risk theory. J. Korean Stat. Soc. 39, 305-314 (2010)

11. Gao, Q., Yang, X.: Asymptotic ruin probabilities in a generalized bidimensional risk model perturbed by diffusion with constant force of interest. J. Math. Anal. Appl. 419, 1193-1213 (2014)

12. Geluk, J., Tang, Q.: Asymptotic tail probabilities of sums of dependent subexponential random variables. J. Theor. Probab. 22(4), 871-882 (2009)

13. Jiang, T., Gao, Q., Wang, Y.: Max-sum equivalence of conditionally dependent random variables. Stat. Probab. Lett. 84, 60-66 (2014)

14. Ko, B., Tang, Q.: Sums of dependent nonnegative random variables with subexponential tails. J. Appl. Probab. 45(1), 85-95 (2008)

15. Li, J.: On pairwise quasi-asymptotically independent random variables and their applications. Stat. Probab. Lett. 83, 2081-2087 (2013)

16. Liu, X., Gao, Q., Guo, E.: Uniform asymptotics for ruin probability of a two-dimensional dependent renewal risk model. Commun. Stat., Theory Methods 45(20), 6045-6060 (2016)

17. Liu, X., Gao, Q., Wang, Y.: A note on a dependent risk model with constant interest rate. Stat. Probab. Lett. 82, 707-712 (2012)

18. Liu, X., Yu, C., Gao, Q.: Precise large deviations of aggregate claim amount in a dependent renewal risk model. Commun. Stat., Theory Methods 46(5), 2354-2363 (2017)

19. Resnick, S.: Extreme Values, Regular Variation and Point Processes. Springer, Berlin (1987)

20. Wang, K., Wang, Y., Gao, Q.: Uniform asymptotics for the finite-time ruin probability of a dependent risk model with a constant interest rate. Methodol. Comput. Appl. Probab. 15, 109-124 (2013)

21. Yi, L., Chen, Y., Su, C.: Approximation of the tail probability of randomly weighted sums of dependent random variables with dominated variation. J. Math. Anal. Appl. 376, 365-372 (2011)

\section{Submit your manuscript to a SpringerOpen ${ }^{\circ}$ journal and benefit from:}

- Convenient online submission

- Rigorous peer review

- Open access: articles freely available online

- High visibility within the field

- Retaining the copyright to your article

Submit your next manuscript at $\boldsymbol{s p r i n g e r o p e n . c o m ~}$ 